\title{
In VANET single Network types i.e. WAVE is not sufficient to provide QoS. UMTS in integration with WAVE can provide efficient network connectivity - A Survey
}

\author{
Nausheen Saeed ${ }^{1}$, Farrukh Nasim², Dr.Riaz ul Amin ${ }^{3}$ Walayat Hussain ${ }^{4}$ \\ ${ }^{1}$ Lecturer, department of computer science, Sardar Bahadur Khan Women University, Quetta \\ ${ }^{2}$ IT coordinator, Save The Children Usa, Quetta Program Office \\ ${ }^{3}$ Associate professor, department of computer science, Balochistan University Of Information \& Technology, Quetta \\ ${ }^{4}$ Assistant professor, department of computer science, Balochistan University Of Information \& Technology, Quetta \\ *Corresponding author E-mail: nausheensaeed1@ hotmail.com
}

\begin{abstract}
This survey provides an insight to the Quality of Service (QoS) provision in Vehicular Adhoc Networks (VANET) through Wireless Access in Vehicular Environment (WAVE) and Universal Mobile Telecommunication System (UMTS). In order to provide ubiquitous and pervasive connectivity for the high mobility vehicular networks in dynamically changing context and service adapting situations, careful techniques must be adopted to provide QoS in VANET applications. To address different implementation scenarios and overcome the shortcomings of WAVE and UMTS, I some alternatives were captured to improve QoS in VANET either by amalgamating WAVE/UMTS or through some other viable protocols.
\end{abstract}

Keywords: Vanet,Adhoc,Qos,UMTS, Wave.

\section{Introduction}

Vanet: vehicular ad hoc are infrastructure less networks step towards making vehicles more intelligent while on roads. The concept of vanet is to make it possible for the vehicles to communicate with each other. Use applications while movie such using VoIP application Google talk or Skype or multimedia streaming more critical applications would be incident alerts or traffic alerts. Makin intelligent machines would increase sale of a particular brand if it incorporates this future trend as well as will make vehicle driving more safety oriented.

Scientists are extensively researching to bring this concept to reality. It is supposed that ambulances firefighting and police will be the first to form a vanet.

\section{UMTS}

Universal mobile telecommunication system infrastructure cellular networks based on SM technology. It works on radio access technology and thus provides more efficiency, bandwidth. And has wider coverage

\section{Vanet QOS requirements}

Vanet QoS are specifically application requirements of certain quality. For example vehicles in the vanet would require getting traffic alerts such as traffic jam/or road accident in certain locality and should be informed so that they can choose an alternate route. The delay sensitive application such as voice over IP and multimedia streaming application would require higher band width. 


\section{Reason for integrating wave and UMTS}

The integration is combining the plus points of both the technologies such as the wider range of UMTS and infrastructure availability which will be a cost effective approach. As well the less expensive wave structure. UMTS infrastructure as is centralized will make vanet communication more secure. And its infrastructure availability does not make the communication dependent on the availability of nodes all these will enhance capability of providing quality of service by vanet.

\section{Quality of service in WAVE}

Based on 802.11p, WAVE exploits the two important channel operations i.e. channel routing and channel selection. The WAVE and 802.11p follow the 802.11e Enhanced Distributed Channel Access (EDCA) QoS model. WAVE further adds the feature of transmitting (WAVE Short Message Protocol) WSMP packets and enforces each WSMP packet should be transmitted using the data rate, channel number and transmission power defined in the packet The WAVE implements channel operations using Channel Coordination Function (CCF). The channel router examines the EtherType field of 802.2 headers to determine the WSMP channel number and routes it to the appropriate queue (Yasser L. Morgan). If it detects an invalid channel number, the packet is simply discarded. The channel selector decides when and how long to monitor a channel and discard the communication if the channel is no longer available. QoS levels are defined by providing different access classes and using the contention window mechanism. Selecting the highest access class is enforced by using the attributes like size of contention window, back off time and size of Arbitrary Inter Frame Spacing. The Lower the number of access class, the higher will be the priority and smaller will be the contention window and AIFS.

\begin{tabular}{|l|l|}
\hline Application & Layer 7 \\
\hline Presentation & Layer 6 \\
\hline Session & Layer 5 \\
\hline Transport & Layer 4 \\
\hline Network & Layer 3 \\
\hline Data Link & Layer 2 \\
\hline Physical & Layer 1 \\
\hline & Fig. 1 \\
IEEE P1609 \\
WAVE \\
IEEE 802.11 \\
DSRC \\
\end{tabular}

WAVE provides specifications, both for physical layer and Media Access Control (MAC) layer, (Kamini). Dedicated Short Range Communication (DSRC), specified under IEEE 802.11P being dedicated to provide QoS at MAC layer. It is an established fact that WAVE can provide QoS with low latency in safety applications such as hazard warnings, vehicle maneuvering and cooperative automatic cruise control (Yunpeng Zang1). If the communication is supported by Road Side Units (RSU), WAVE can fulfill the requirements of safety and non-safety applications.

Most of WAVE deployments use frequency spectrum of $75 \mathrm{MHzband}$ at $5.9 \mathrm{GHz}$, divided into 7 channels, each operating at $10 \mathrm{MHz}$, allowing a high data transmission rate of 6 - $27 \mathrm{Mbps}$ and short range communication in proximity of 300 meters. Later on, in 2008, 30MHz frequency has also been approved for use in Europe.

\section{Quality of service in UMTS}

Universal Mobile Telecommunication Systems (UMTS) is an improved version of $3 \mathrm{G}$ and is a step ahead of regular cellular communication technology. It operates at a frequency of $2 \mathrm{GHz}$ and allows a peak data rate of $2 \mathrm{Mbps}$ and 384 Kbps for upload and download. The UMTS High Speed Data Packet Access offers the data transmission rate up to 7.2 Mbps/ 2Mbps (Tarik, Abderrahim). UMTS widens the communication range of the network typically ranging from 8 to $10 \mathrm{Km}$ per base station.

UMTS introduces a concept of "Bearer Service" that ensures the Quality of Service in all aspects such as control signaling, user plane transport and QoS management functionality. It addresses the QoS challenges by defining four types of traffic classes for different categories of applications (Emanuel, Gabriel) as summarized in the table below: 
Table 1

\begin{tabular}{ccc}
\hline Traffic classes & Characteristics & Application \\
\hline $\begin{array}{c}\text { Conversational } \\
(\mathrm{CO})\end{array}$ & Low delay, low jitter, symmetric traffic, no buffering speech, VoIP, & Video \\
$\begin{array}{c}\text { Streaming (ST) } \\
\text { Interactive (IN) }\end{array}$ & $\begin{array}{c}\text { Moderate delay, moderate jitter, asymmetric traffic, buffering allowed video } \\
\text { streaming, }\end{array}$ & Audio streaming \\
$\begin{array}{c}\text { Moderate jitter, asymmetric traffic, buffering allowed, request response } \\
\text { pattern }\end{array}$ & Web browsing \\
Background (BK) & $\begin{array}{c}\text { destination doesn't expect data within a certain time, preserve payload } \\
\text { content, asymmetric traffic, buffering allowed }\end{array}$ & $\begin{array}{c}\text { Email, file } \\
\text { downloading }\end{array}$ \\
\hline
\end{tabular}

Quality of Service requires agreed mechanisms for traffic bearers in local bearer, UMTS bearer and external bearer domains. In UMTS Bearer QoS, the Serving GPRS Support Node (SGSN) is responsible for controlling UMTS Bearer service QoS, which is composed of two important components.

The Radio Access Bearer (RAB) is a combination of Radio Bearer (RB) and Iu bearer that require radio resources over the air. The Iu bearer service requires QoS for IP and ATM transport.

Backbone Bearer QoS is an IP based packet switched network implemented within the Core Network. It inherits the IP QoS techniques such as Differentiated Services (DiffServ) or Resource Reservation Protocol (RSVP).

UMTS defines how UMTS Bearer QoS mechanisms incorporate with the IP QoS mechanisms

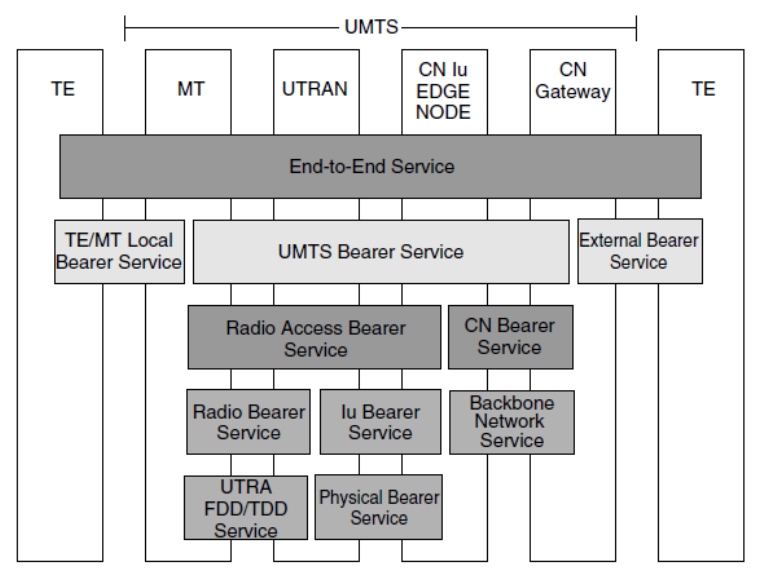

Fig. 2: Architecture of UMTS bearer service

UMTS bearers have to be generic by nature (Jouni, Antti) in order to allow compatibility with the existing application and future extensibility to accommodate the evolving technologies.

\section{Is WAVE sufficient to provide QoS in VANET?}

WAVE is aimed at providing support to vehicular movement at low speeds and closer proximity of about $300-500$ meters through WLAN at $2.4 \mathrm{GHz}$ although it can deliver required QoS to the applications but needs alternatives to deal with transmission over longer distances.

WAVE is prone to external interference and suffers with increased number of nodes in a given area. Many other radio devices such as cordless phones, mobile devices, computers etc. can further add to the congestion and force the communicating vehicular devices to frequently switch their channels. If the given channels get busy, the communication and information processing delays could become unreasonably large and pose serious question mark to the safety critical applications.

WAVE relies on a contention based medium access method i.e. CSMA/CA and by design, it has to avoid collision in communication. If the number of users increases in a given area, the chances of cross channel interference and collision also rise. Based on this principal I assume that the QoS in WAVE heavily relies on the number of nodes participating in the ad hoc network. It also suffers from Near-far disturbance problem, i.e. the critical safety messages from the remote vehicle may not reach us due to near end interference.

The Quality of Service Channel Coordination Function (CDF) heavily relies on the time synchronization source in the WAVE channel selection. If the Coordinated Universal Time (UTC), which is usually provided through Global 
Positioning System, becomes unavailable, the entire communication may collapse in absence of an alternate time keeper.

In addition to the time source and channel selection problem, WAVE also doesn't make an effective use of the frequency spectrum. The source and control channels are bifurcated strictly that may cause a contention situation when all the available control channels are occupied and a safety message from a distant vehicle arrives.

WAVE may not necessarily suit all deployment scenarios and road conditions. An example is of a long highway with very fast but little number of vehicles. The vehicles may not communicate to each other due to long intra-vehicle distances and if we plan to deploy the RSUs, it will lead to a huge infrastructural cost

\section{Can UMTS in integration with WAVE provide efficient network connectivity?}

As we can see a number of challenges posed on VANET by the native design of WAVE, we need to redesign WAVE or opt for a better alternative that can fully complement or supplement WAVE to improve QoS it in the given scenarios

1) UMTS networks are built using well defined infrastructure that provides coverage over large distance. Taking further advantage of the infrastructure we can deeply integrate authentication, high mobility and security. On the other hand, the transmission delay is increased as there is no direct communication among the mobile nodes and every message has to take its route through the central infrastructure. This leads us to an amalgamation of WAVE and UMTS; WAVE to be used over shorter distances \& direct communication, while UMTS for high mobility and transmission over longer distances.

2) Although the transmission delay in UMTS is greater than WAVE in general, but use of infrastructure allow $\mathrm{s}$ multiple nodes to communicate to a group of other vehicles simultaneously. In WAVE, the CSMA/CA strictly disallows simultaneous transmissions and the number of participating vehicles adversely affected the channel capacity, while in UMTS the dedicated bandwidth and ability to simultaneously multicast messages over longer distances through fixed infrastructure is an added advantage.

3) Admission control in UMTS networks support real-time and ordinary traffic whereas transport mechanism of WAVE is best-effort.

4) In UMTS, bearer parameters are negotiated such as bearer type, bit rate, bit error rate (BER), transfer rates etc. (Maciej, Ziemowit and Marcin) These parameters can be negotiated on a new connection or during handing off existing connections. The bearers may be scheduled or even pre-empted for specific type of traffic and are flexible to adapt to varying network conditions such as status of link, available bandwidth and quality of radio.

5) Keeping in view the characteristics of WAVE and UMTs, one can easily derive an idea of integrating both services under the same hood to achieve QoS in vehicular networks over short and long distances while implementing better techniques of channel utilization.

\section{Integrating UMTS into WAVE}

Since UMTS and WAVE are complementary to each other in terms of technique, infrastructure, frequency, performance, range and cost, it is practically infeasible to merge both into a single technology or protocol. Most of the researches have therefore suggested hybrid models to enjoy the features of both WAVE and UMTS. A typical integration model may comprise of using WAVE for close proximity communication or dealing with the low latency and local hazard warning applications; while using UMTS for longer distances or effectively utilizing the communication channels to provide QoS. (Yunpeng Zang, Bing Han) GPS devices can be used for acquiring location information and time synchronization

\section{Approaches to integrate UMTS into WAVE}

Several studies and research works have been out there to propose integration approaches for UMTS and 802.11 based vehicular networks. Few of them are listed below: 


\subsection{An architecture for integrating UMTS and 802.11 WLAN networks}

Under this approach, the low cost extensions of UMTS and 802.11 LANs are deployed to cover the hot spots. The data is maintained via best-effort approach through WAVE and UMTS takes care of the voice in parallel. This model defines two commonly used scenarios:

1) Roaming Scenario, in which WAVE and UMTS are not connected to each other, however a user may subscribe to any required network if both networks are operated by the same service provider.

2) Hotspot Scenario, where the UMTS operators allow robust connectivity in specific areas called hotspots.

The mobile nodes can access both networks provided that they are equipped with dual interfaces, one connected to 802.11 networks and the other to the UMTS

The UMTS and WAVE networks could be joined through IPv6 backbone, UMTS authentication infrastructure, storing user data in SIM card or through any viable connection (Muhammad Jaseemuddin).

\subsection{An efficient routing protocol for connecting vehicular networks to the internet}

Here, a new protocol was designed that utilizes the attributes of vehicular movement and forecast the future behavior of the vehicles. Based on the perceived vehicle movements, the vehicle is connected to the wired network via a route with maximum possible lifetime. The routing protocol then spreads the advertisement messages without flooding the vehicular network and performs seamless handovers.

The gateway discovery process is limited to a predefined geographical zone and only few relays are allowed to rebroadcast the advertised messages. The transmission range and the future locations of the vehicles can then be forecasted through stability matrix (Abderrahim).

\subsection{An intelligent agent-based scheme for vertical handover management across heterogeneous networks}

This technique incorporates an intelligent and adaptive agent in the vehicles and access points to perceive the environment and then the vertical handovers are done for the best available network using a knowledge plane. It is important to note that several access methods are combined here using IP as glue and the agents in the network devices are autonomous, adaptive and distributed in nature (Atiq Ahmed).

\subsection{Dynamic clustering-based adaptive mobile gateway management in integrated vanet 3G heterogeneous wireless networks}

Dynamic clustering is yet another approach to form a group of vehicles according to different metrics. This clustering could take place on the basis of direction of movement, UMTS signal strength, IEEE 802.11p wireless transmission range, TTL value of a cluster, and so on (Abderrahim)

By operating more than one gateway, bottlenecks and congestion can be treated more effectively.

\section{Critical points of integration approaches}

The research literature on the subject has undoubtedly made a great contribution in achieving a better vehicular environment by combining the features of WAVE and UMTS but has also highlighted more areas to be explored while leaving a point of criticism in their own proposed techniques. Few important points are mentioned below:

1) WAVE greatly suffers from the collisions at MAC layer. This Is due to synchronization back off processes for the channel selection and switching. Control channels or the high priority messages also suffer from these collisions as the back off windows are small in 802.11P and the Distributed Coordination Function (DCF) relies on RTS/ CTS mechanism to share the media.

2) In order to provide seamless and reliable end to end connectivity, the underlying network architecture must ensure the required quality of service. UMTS, although being way ahead than WAVE can still only ensure quality of service for delay-sensitive traffic if and only if it is working in Radio Resource Control (RRC) mode.

3) The areas unsuitable for WAVE must assume UMTS connectivity in the entire area. This could lead to serious issues of handoff \& service continuity if there are UMTS dead spots on the way.

4) All integration approaches proposed using two radios for communication. This could have been simplified by using dual band network interface cards. Same could go for the communication equipment, i.e. using a dual-band 
communication infrastructure will not only elevate several challenges of WAVE but also provide a better configuration for UMTS or other $3 \mathrm{G}$ technologies.

5) A mobile node can connect to a single network only at a given time. It means that the nodes often skip the chances of availing the potential bandwidth available in WAVE or UMTS network.

6) The time source in all approaches referred to a GPS device. This overhead could have been taken off by allowing UMTS to be used as a time source.

7) Currently GPS is the only navigation system referred to in the available literature. More research needs to be carried out to incorporate the upcoming standard such as GLONASS.

8) It is always claimed that the vertical handoff is or has to be seamless; the techniques are however not mentioned.

9) Performance analysis seems to be a grey area in the research material of integration approaches. One only finds it in the "future work" section of the paper. It is also possible that the simulation results deviate from the actual deployment scenarios.

10) Admission control also needs a great attention in context of resource optimization, bandwidth contention and privacy.

11) The integration approaches, QoS and routing protocols deal with data and applications. No mechanism was found to prioritize the actual traffic according to the type of vehicles such as ambulances, firefighting vehicles, school vans, police escorts, etc.

12) Every paper defines a new protocol structure or some additional overhead to the existing protocols. Had it been better if researchers opted for industry standard protocols that could provide the quality of service in the respective areas such as MPLS, WWAN, WiMAX, EVDO, LTE etc

\section{Conclusion}

After comparing all the pros and cons of WAVE and UMTS, despite all the criticism, our interest still remains to ensure availability and quality of service in the vehicular network before, during and after handoffs. It is also important to note that WAVE and UMTS complement each other in certain scenarios and can improve service availability and graceful handoffs in most of the scenarios where adequate infrastructure is available; however UMTS can still not provide the quality of service for life critical safety applications. Future consideration for VANET applications must be the QoS models, QoS signaling mechanism, QoS routing protocols and QoS medium access methods so as to ensure stateful service availability, service migration/ handoff and reliability in dynamically changing contexts.

\section{References}

[1] IETF RFC2386 | http://www.ietf.org/rfc/rfc2386.txt | accessed on 21 Feb 2013, 8:23 PM

[2] An Architecture for Integrating UMTS and 802.11 WLAN Networks | Muhammad Jaseemuddin | Proceedings of the Eighth IEEE International Symposium on Computers and Communication (ISCC'03) | page 1530-1346/03

[3] An efficient routing protocol for connecting vehicular networks to the Internet | Abderrahim Benslimane, Saman Barghi, Chadi Assi | Pervasive and Mobile Computing 7 (2011) | page 98-113

[4] An intelligent agent-based scheme for vertical handover management across heterogeneous networks | Atiq Ahmed, Leïla Merghem, Boulahia, Dominique Gaiti | Ann. Telecommun. (2011) 66:583-602 | DOI 10.1007/s12243-010-0235-6

[5] Design Guidelines for a Network Architecture Integrating VANET with 3G \& Beyond Networks | Tarik Taleb and Abderrahim Benslimane | NEC Europe Ltd., Germany, University of Avignon, France, 978-1-4244-5637-6/10/\$26.00 @2010 IEEE

[6] Dynamic Clustering-Based Adaptive Mobile Gateway Management in Integrated VANET - 3G Heterogeneous Wireless Networks | Abderrahim Benslimane, Senior Member, IEEE, Tarik Taleb, Senior Member, IEEE and Rajarajan Sivaraj | IEEE Journal on Selected Areas in Communications, vol. 29, no. 3, March 2011 | d.o.i: 10.1109/JSAC.2011.110306 | page 559-570

[7] Hybrid Solution for Vehicular Communications | Yunpeng Zang1*, Bing Han1, Sabine Sories2, Guido Gehlen2

[8] Managing DSRC and WAVE Standards Operations in a V2V Scenario | Yasser L.Morgan | International Journal of Vehicular Technology doi | 10.1155/2010/797405 | page 1-18

[9] Modeling and Simulation of WAVE 1609.4-based Multi-channel Vehicular Ad Hoc Networks | Ali J. Ghandour, Marco Di Felice, Hassan Artail

[10] QoS Support in UMTS Networks, Performance Evaluation and Perspectives towards an Autonomic Resource Management Emanuel Puschita, Gabriel Manuliac, Tudor Palade, Alexandru Caruntu | AFIN 2011 : The Third International Conference on Advances in Future Internet | ISBN: 978-1-61208-148-9 | page 25-30

[11] UMTS Services and Applications | Jouni Salonen, Antti Toskala and Harri Holma, 2002 | John Wiley \& Sons, Ltd | Page 11-29

[12] Understanding UMTS Radio Network Modelling, Planning and Automated Optimisation | Edited by Maciej J. Nawrocki, Mischa Dohler and A. Hamid Aghvami | @ 2006 John Wiley \& Sons, Ltd

[13] VANET Parameters and Applications: A Review, Kamini1 Rakesh Kumar2, Global Journal of Computer Science and Technology |P a g e 72 Vol. 10 Issue 7 Ver. 1.0 | September 2010

[14] Wireless Access for Vehicular Environments | Bo Li, Mahdieh Sadat Mirhashemi, Xavier Laurent, Jinzi Gao. 